\title{
Ionic liquids at charged surfaces: Insight from molecular simulations.
}

\author{
Dmitry Bedrov*, Jenel Vatamanu, and Zongzhi Hu \\ Department of Materials Science \& Engineering \\ The University of Utah \\ 122 S. Central Campus Dr., Rm. 304 \\ Salt Lake City, UT 84112
}

\begin{abstract}
Understanding of molecular level structure and mechanisms of the formation of electric double layers in realistic ionic liquid-based electrolytes on charged electrode surfaces is one of scientifically and technologically key areas that has attracted a lot of attention over the last decade. Extensive experimental, theoretical, and modeling studies have been dedicated to this challenging topic in order to establish fundamental correlations between the details of molecular structure of electrolyte and the properties of the electric double layers (EDL) forming on various electrodes. While great progress has been made in advancing our understanding of EDL properties and their influence on the performance of supercapacitors, batteries, and other energy storage devices, there are still a number of challenges and controversies that have not been resolved. In this manuscript, we demonstrate how atomistic molecular dynamics simulations provide a powerful tool for dealing with these challenges and can facilitate the design of novel materials for advancing energy storage technologies.
\end{abstract}

Corresponding author: Dmitry Bedrov, e-mail: d.bedrov@utah.edu 


\section{Introduction.}

The behavior and properties of room temperature ionic liquids (RTILs) on charged electrode surfaces have attracted significant attention in the last decade due to potential promise for utilization of the RTILbased electrolytes for application in the new generation of energy storage devices. A particularly significant progress in the application of RTIL-based electrolytes has been achieved in electric double layer (EDL) capacitors (EDLCs), also known as super- or ultracapacitors. In these devices, the charge/energy is stored through the rearrangement and accumulation of ions in a couple nanometers thick layer of electrolyte at high specific surface area electrodes [i]. EDLCs have great potential to complement (or replace) batteries in electrical energy storage, particularly when the high power density and fast power delivery/uptake and long cycling life are required. In purely capacitive mode, the absence of Faradic reactions during charge and discharge cycles eliminates the phase changes in the active electrode material that typically occur in batteries [ii]. EDLCs can therefore, in principle, sustain millions of cycles. Substantial efforts have been dedicated to improve the energy density of EDLCs through the design of nanostructured electrodes with a high specific surface area and gaining fundamental understanding of the interplay between various phenomena defining the performance of these devices. Realization that RTILs are promising candidates as electrolytes for high energy density capacitors has opened new routes for optimizing the performance of EDLCs. RTILs are in general electrochemically and thermally more stable than conventional electrolytes, allowing for higher charging voltages.

In order to further improve the performance of EDLCs, a more advanced understanding of charge storage mechanisms and their correlations with the chemical structure and composition of electrolytes are needed. This understanding is crucial for further development of novel advanced nanostructured electrodes and electrochemically stable electrolytes. However, our understanding of correlations between properties of different EDLC components and their influence on the EDLC performance is still immature. The progress in this fundamental understanding is hindered by the complex interplay of several molecular scale phenomena that make experimental studies of EDLs quite challenging and often resulting in inconsistent 
data. For example, Figure 1 shows a comparison of several reported in the literature experimental data for differential capacitance (DC) obtained on gold, platinum, and glassy carbon electrodes for the same (or very similar) RTILs [iii-vii]. The DC is one of the key properties used to characterize structural changes in the EDL as a function of electrode potential as well as a direct measure of the energy stored by a given electrode/electrolyte combination. As we can see from Figure 1, the shape and the magnitude of DC reported by different studies can significantly deviate from each other. The magnitude of DC from two different measurements can be different up to a factor of five, particularly at low potentials. Note that the inconsistencies shown in Figure 1 were obtained for the simplest electrode/electrolyte set up geometry, i.e., flat electrode surfaces in contact with bulk electrolyte. Needless to say that interpretation of experimental results become even more complicated for the technologically more practical nanoporous electrodes, where electrolyte EDL layers are forming in nanopores with very heterogeneous pore size, shape, and surface structure distributions [viii,ix]. In these electrode materials, electrolyte is subjected to nanoconfinement, local curvature, and atomic scale roughness of exposed electrode surface, all of which can significantly influence the magnitude and dependence of DC [x].

Several theoretical models have focused on the prediction and explanation of correlations between the EDL structure and the dependence of DC as a function of electrode potential [xi-xxvii]. These models found that on a flat surface, in general, the DC can be either a camel-shaped with a minimum near the potential of zero charge (PZC) or a bell-shaped with a maximum at low potentials. The camel-shape DC typically has a low-voltage minimum (the U-shape region) flanked by two maxima at higher electrode potentials. These dependencies were explained by the analytical model of Kornyshev [xxviii] and attributed them primarily to steric exclusion effects. Specifically, on surfaces where at PZC the EDL structure has a relatively low density of ions and, hence, can pack more ions without significant free energy penalty, the rate of electrode charge $(\sigma)$ accumulation as a function of electrode potential $\left(\mathrm{U}_{\text {electrode }}\right)$ is high. Therefore the DC, defined as $\mathrm{DC}=\mathrm{d} \sigma / \mathrm{dU}_{\text {electrode }}$ is increasing with the increase of electrode potential. However, at higher potentials, the electrode surface eventually becomes overcrowded with 
counterions and $\mathrm{d} \sigma / \mathrm{dU}_{\text {electrode }}$ (i.e., DC) begins to decrease with further increase of $\mathrm{U}_{\text {electrode. }}$ Therefore, on surfaces that at PZC have loosely-packed electrolyte the camel-shape DC is expected. In contrast, if at PZC the EDL is already crowded by the counterions and/or has a high overall density due to van der Waals interactions of electrolyte with the electrode surface, i.e., the EDL is sufficiently incompressible, a further increase in applied potential will not lead to a sufficient increase of electrode charge to allow an increase in DC and, hence, a bell-shape DC dependence can be expected.

Recent experiments [iii-vii,xxix,xxx] showed that both types of DC are observed in RTIL electrolytes. While many of these experimental data can be qualitatively understood/interpreted within the framework of theoretical models mentioned above, a careful examination of several recent experimental studies showed an apparent inconsistency between them (as shown in Figure 1) as well as a qualitative disagreement with predictions of basic theoretical models. For example, Su et al. [iii] studied 1-butyl-3-methylimidazolium $\left(\mathrm{C}_{4} \mathrm{MIM}\right)$ cation-based RTIL, $\left[\mathrm{C}_{4} \mathrm{MIM}\right]\left[\mathrm{BF}_{4}\right]$, on $\mathrm{Au}(001)$ electrode and reported a bell-shape DC with a surprisingly large maximum near PZC $\left(\sim 25 \mu \mathrm{F} / \mathrm{cm}^{2}\right)$. In contrast, for a very similar electrolyte $\left[\mathrm{C}_{4} \mathrm{MIM}\right]\left[\mathrm{PF}_{6}\right]$ Alam et al. $\left.{ }^{[\mathrm{iv}}\right]$ found essentially a constant $\mathrm{DC}\left(\sim 6-7 \mu \mathrm{F} / \mathrm{cm}^{2}\right)$ as a function of electrode potential. Similarly large differences in DC were observed for $\left[\mathrm{C}_{4} \mathrm{MIM}\right]\left[\mathrm{PF}_{6}\right]$ on glassy carbon electrode as measured by Lockett et al. [vi] who reported DC fluctuating between 4 and $8 \mu \mathrm{F} / \mathrm{cm}^{2}$ and Silva et al. [v] who obtained DC in the $18-27 \mu \mathrm{F} / \mathrm{cm}^{2}$ range.

Another controversial issue in EDL properties of RTIL-based electrolytes is the temperature dependence of EDL structure and DC. A number of experimental studies, such as Lockett et al. [vi,xxxi], Silva et al. [" $\left.{ }^{\mathrm{v}} \mathrm{xxxii}\right]$, Alam et al. [xxxiii-xxxv] and Siinor et al. [xxxvi] have reported a systematic increase of DC with increasing temperature for commonly studied RTIL-based electrolytes comprised of alkylimidazolium $\left(\mathrm{C}_{\mathrm{n}} \mathrm{MIM}\right)$ cations and $\mathrm{BF}_{4}^{-}, \mathrm{PF}_{6}^{-}$or Bis(trifluoromethanesulfonyl)(TFSI) anions on several electrode surfaces (Au, Pt, and glassy carbon). Surprisingly, in some of those studies the capacitance magnitude showed a significant increase with increasing temperature even at large electrode potentials where the electrode surface is expected to be crowded with electrolyte ions and the EDL 
structure and composition to be only weakly dependent on temperature. For example, Silva et al. showed almost a factor of two increase in capacitance across a wide range of voltages and within a 55-degree temperature window [v]. In contrast, Druschler et al. [xxxvii] found that the capacitance due to the fast relaxation process, that was associated with electrolyte reordering in the EDL, had an opposite temperature dependence. Specifically, that work reported DCs ranging between $4-7 \mu \mathrm{F} / \mathrm{cm}^{2}$ in the investigated electrode potential window and an overall small decrease of DC with increasing temperature [xxxvii]. Alam et al. also reported only weakly changing DC with temperature for $\left[\mathrm{C}_{2} \mathrm{MIM}\right]\left[\mathrm{BF}_{4}\right]$ on $\mathrm{Hg}$ electrode [xxxviii]. A very recent experimental work of Cannes et al. on $\left[\mathrm{C}_{4} \mathrm{MIM}\right][\mathrm{TFSI}]$ has also showed an overall decrease of DC with increasing temperature for several electrode surfaces [xxxix].

Taking into account the outstanding issues discussed above, the vast amount of possible anion-cation combinations that can comprise an RTIL-based electrolyte, and the fact that detailed experimental characterization of a couple nanometer-wide EDLs formed in these electrolytes is very challenging, it is clear that both experimental and theoretical investigations of these systems require a complementary atomistic scale simulation methods that can help to sort out the complexity of molecular level phenomena occurring in these systems. Below we discuss several aspects of such simulations and discuss the key insights obtained from investigation of RTIL-based electrolytes on charged electrode surfaces using atomistic molecular dynamics (MD) simulations.

\section{Methodology.}

Electrode treatment. Conceptually, molecular dynamics (MD) simulations of electrolytes near charged surfaces should be a perfect tool for studying the EDL structure and properties, particularly if atomistic force fields are employed and, hence, the specific details of electrolyte chemical structure can be taken into account. The latter includes variations in the ions' size, shape, and conformations as well as the details of partial atomic charge distributions and induced polarization for a variety of molecular architectures that are used to comprise RTILs. In practice, there are number of challenges that MD simulations are facing in the modeling of these systems. Besides the obvious dependence of the quality of 
simulation predictions on the accuracy of employed force fields, which is an issue for all atomistic MD simulations independently of what systems are investigated, there are couple specific issues related to the modeling of RTILs on charged surfaces. In this section we briefly discuss those issues.

The first issue is related to how in MD simulation the charged surface is coupled with the rest of the system. The most straightforward approach is to confine electrolyte between two solid surfaces and then apply constant charges $+Q$ and $-Q$ on the electrodes. The electrode charge is usually homogeneously distributed between the electrode surface atoms. As the simulation progresses, electrolyte will rearrange near each charged surface and the corresponding EDLs would form. The electrode potentials as well as the potential difference between electrodes in this simulation scheme are not known a priori and have to be determined by analyzing the average charge density profiles established in electrolyte in the direction perpendicular to the surface. This approach is employed in the overwhelming majority of simulation works related to RTILs (and other electrolytes) on charged surfaces. The advantage of this approach is its simplicity and therefore it can be easily implemented using available molecular simulation packages. However, there is an important drawback in the underlying physical assumption of this approach compared to realistic applications. In real capacitors, batteries, or any other storage device the controled parameter is the electrostatic potential difference between electrodes, not the amount of charge on each electrode as assumed in these simulations. In realistic system, the charge on the electrode surface is building up in response to EDL restructuring and electrode polarization associated with this restructuring.

Therefore a more natural simulation set up would be a methodology that allows simulation of electrolyte confined between two electrodes with a fixed applied potential difference between electrodes. In contrast to the constant electrode charge approach discussed above, the constant applied potential method does not assume a priory any charge distribution on the electrode surface. Instead, it constrains the potential difference between two electrodes (see Figure 2a.) and allows charges on the electrode surfaces to equilibrate minimizing the electrostatic energy of the system using an iterative procedure [xl,xli,xlii]. In this approach while partial atomic charges of electrolyte molecules are fixed, the charge on any given atom 
of electrode surface is not. Every time step (or few time steps depending on the system) the iterative procedure of minimizing electrostatic energy provides new distribution of charges on the electrode surface. Therefore, the charge of individual atoms on the electrode can a) evolve in response to local EDL restructuring, i.e., due to electrode electronic polarization from electrolyte rearrangement near the surface, and b) have any spatial distribution in accordance with the structural heterogeneities (surface roughness or defects) inherent to a given electrode surface structure. The latter aspect, i.e., the inhomogeneous charge distribution on the electrode surface, is key in correctly capturing the EDL structure and DC on nanostructured surfaces. This is clearly illustrated in Figure 3, where the charge distributions on basal and prismatic plane graphite surfaces are shown as obtained from our MD simulations using the constant applied potential approach. Unlike, the constant charge method that would use the delta function distribution, relatively broad distributions of charges for the electrode surface atoms are observed for both surfaces during simulations. Despite the fact that the atomic scale roughness of the prismatic plane graphite is very small, the depth of the atom layers offset is just $1.41 \AA$, the charge distribution for the electrode surface atoms is significantly different and more complex compared to the one observed on atomically flat basal plane graphite. Therefore, while for atomically flat surfaces representing the relatively symmetric charge distributions (Fig. 3a) by a single average value of the charge for all electrode surface atoms might be a reasonable approximation, for the atomically rough surfaces ( e.g., the prismatic graphite) such approximation clearly cannot be adequate. Taking into account that in many energy storage devices various chemical modifications of electrode surfaces are considered as one of the possible routes for the electrode material design [xliii] the ability of MD simulations to capture the details of charge distribution on electrode surfaces, therefore reflecting the intrinsic characteristics of the surface structure, will be crucial for the ability to predict accurate EDL properties. Finally, if the evolution of the EDL formation or the dynamics of charging/discharging processes are of interest, the constant applied potential approach is a must as it provides the correct coupling between EDL structure evolution and the charge accumulation on electrodes. This has been clearly demonstrated in recent simulations of Merlet et al. [xliv] 
showing that modeling of RTIL electrolyte insertion inside nanoporous electrodes using the constant charge approach will create sever artifacts compared to the constant applied potential approach.

While several previous works have demonstrated that the constant applied potential approach is the appropriate methodology to model EDL restructuring on nanostructured electrodes, it still requires further enhancement to accurately model more realistic electrode structures and materials. Specifically, so far, this methodology have assumed that the electrode material is purely conductive. However, many realistic carbon based materials such as CDC or activated carbon contain a variety of heterogeneous 1D and 2D structural components, edges and defects that affect local electronic conductivity. Therefore, these materials cannot be treated as ideal electron conductors and the semiconducting character of these materials has to be taken into account. Further enhancement of this methodology using the information obtained from $a b$ initio calculations is needed to extend MD simulations for modeling of materials with heterogeneous electronic conductivity [xlv].

Simulation analysis. Since most of the results discussed in this paper will be from simulations using the constant applied potential method, here we briefly review definitions of properties used in the analysis and how they are extracted from such simulations. The EDL potential (or the potential drop within electrode/electrolyte interfacial region), $\mathrm{U}_{\mathrm{EDL}}$, is defined as the difference between electrostatic potential on the electrode surface $\left(\phi_{\text {electrode }}\right)$ and the potential in bulk electrolyte $\left(\phi_{\text {bulk }}\right)$ (see Figure 2$)$,

$$
\mathrm{U}_{\mathrm{EDL}}=\phi_{\text {electrode }}-\phi_{\text {bulk }}
$$

The electrode potential $\left(\mathrm{U}_{\text {electrode }}\right)$ is defined as the EDL potential $\left(\mathrm{U}_{\mathrm{EDL}}\right)$ relative to the potential of zero charge (PZC).

$$
\mathrm{U}_{\text {electrode }}=\mathrm{U}_{\mathrm{EDL}}-\mathrm{PZC}
$$

The PZC is computed as the potential drop within the EDL for uncharged electrode surfaces and can be obtained from simulation where the potential difference between two electrodes is set to zero. Therefore, while the total potential difference between electrodes $\Delta \mathrm{U}$ is fixed the potentials on individual electrodes only have to satisfy constrain that $\Delta \mathrm{U}=\left|\mathrm{U}_{\text {electrode+ }}\right|+\left|\mathrm{U}_{\text {electrode- }}\right|$ and, therefore in principle, do not have to be 
symmetric. In reality, for most flat electrode geometries the individual electrode potentials are very close to be equal in magnitude and only differ by sign.

From simulation trajectories the electrode and electrolyte charge density profiles in the direction perpendicular to the electrode surfaces (for convenience we defined this as the $z$-axis) can be computed and then used to calculate the Poisson potential by integrating the $1 \mathrm{D}$ Poisson equation along the $z$ direction:

$$
\nabla_{\mathrm{z}}\left[\varepsilon_{0}\left(\nabla_{\mathrm{z}} \phi(\mathrm{z})\right)\right]=-\rho(\mathrm{z})
$$

The DC is then computed using the numerical derivative of the electrode charge density per unit surface with respect to electrode potential.

$$
\mathrm{DC}\left(\mathrm{U}_{\text {electrode }}\right)=\mathrm{d} \sigma / \mathrm{d} \mathrm{U}_{\text {electrode }}
$$

Note that in this definition, the capacitance is normalized per unit surface area of the electrode surface. In order to numerically differentiate Eq. (2) we followed the procedure originally described in Ref. xlvi in which several (typically five or seven) consecutive points of $\sigma\left(\mathrm{U}_{\text {electrode }}\right)$ dependence within $0.3-0.5 \mathrm{~V}$ potential window are approximated with a parabola and the DC in the middle point is obtained using an analytical derivative of the fitted parabola.

Finally, we would like to point that in our simulations all electrodes are treated as conductors. While for some electrodes, such as e.g. gold or platinum, this assumption is natural, other electrodes, such as e.g. carbon-based electrodes, can have a noticeable semiconducting character. ${ }^{\text {xlvii }}$ The latter can significantly vary depending on electrode preparation and fabrication conditions. As was shown by Gerisher et al. [xlvii], in semiconductive electrodes, a space charge distribution inside the electrode is generated and the total capacitance $(\mathrm{C})$ is given by the contribution of the capacitance inside the semiconductor $\left(\mathrm{C}_{\mathrm{sc}}\right)$ and the capacitance generated by electrolyte ordering $\left(\mathrm{C}_{\mathrm{EDL}}\right)$ near the electrode surface: $1 / \mathrm{C}=1 / \mathrm{C}_{\mathrm{sc}}+1 / \mathrm{C}_{\mathrm{ELD}}$. The contribution of $\mathrm{C}_{\mathrm{sc}}$ to the total $\mathrm{DC}$ for semiconductive electrodes is not negligible and a U-shaped DC measured on graphite was previously interpreted by Gerischer as a result of changes in the electronic density of states and the Fermi distribution in pyrolitic graphite itself rather than 
due to electrolyte ordering near the electrodes. Similar trends were found in recent modeling by Kornyshev et al. $\left.{ }^{\mathrm{xlv}}\right]$ who effectively included the semiconducting nature of graphite materials in their simulations. The simulations that will be discussed below assume that graphite-based electrodes are purely conductive and therefore can only access the capacitances given by the electrolyte ordering/restructuring near the surface (i.e, the $\mathrm{C}_{\mathrm{EDL}}$ ).

\section{Results and Discussion.}

Atomically flat surfaces. We begin with a brief summary of simulations of different RTILs on what we refer as atomically smooth electrode surfaces. These include defect free metal surfaces such as e.g. gold or graphite electrodes with the basal plane exposed to electrolyte. A comparison of DC obtained on such surfaces for variety of RTILs results in DC with very similar magnitudes and that have only a weak dependence on electrode potential. This is illustrated in Figure 4 where DCs obtained for RTILs comprised of various cations and anions on gold and basal plane graphite surfaces are shown as function of electrode potential. All systems show that in the electrode potential window between $\pm 2.5 \mathrm{~V}$ (corresponding to the applied potential difference of $4-5 \mathrm{~V}$, which is a typical range of interest for the

current practical applications of these electrolytes) the DC magnitude varies between 4 and $6 \mu \mathrm{F} / \mathrm{cm}^{2}$. These values are in a good agreement with a number of experimental data reported in the literature, such as e.g. refs. [iv,vi,xxxvii,xxxix], yet in a noticeable disagreement with those experiments that show significant, i.e. a factor of several, changes in DC as a function of electrode potential (e.g., refs [iii,v,xxxii,xxxiv,xxxvi]). Of course the latter is not surprising taking into account the inconsistencies between different experimental data discussed above.

Despite a relatively weak dependence of DC on the electrode potential, we can clear distinguish that some systems show a well-defined camel-shape dependence while others are more indicative of a bellshape, consistent with theoretical model predictions mentioned above. In order to understand the details and correlations of the changes in DC with electrode potential we have to analyze the changes in EDL 
structure. The latter is usually characterized by the ion center-of-mass or atomic density profiles as a function of separation from the electrode surface. An example of such density profiles is shown in Figure 5 for $\left[\mathrm{C}_{4} \mathrm{MIM}\right]\left[\mathrm{PF}_{6}\right]$ RTIL on Au electrode. These profiles can clearly identify the rearrangement of co- and counter-ions in the EDL as a function of electrode potential. We can also see that the extent of structural perturbations in RTIL near charged electrodes is quite short, all density oscillations diminish within 2-3 nms from the electrode surface.

The EDL restructuring in RTILs is often accompanied with a noticeable reorientation of ions that are not spherically symmetric. In these molecular liquids, where the distribution of partial atomic charges in each ion type depends on electro negativity of atoms and their local chemical environments, the reorientation of ions can significantly contribute to the charge rearrangement near the surface and, therefore, can influence the EDL capacitance without significant displacement of ions' center-of-mass. This point is illustrated in Figure 6 where the changes in the charge distribution in the electrolyte interfacial layers of different width $d$ have been analyzed and compared with the DC dependence on electrode potential. Specifically, for each system we have defined an interfacial layer with several different widths ranging from 5 to $20 \AA$ near the electrode surface and have calculated the cumulative charge in the defined layers. Then the cumulative charge obtained from simulations at different applied potentials was differentiated with respect to electrode potential. If the excess charge in the selected electrolyte layer is equal in magnitude (opposite in sign) to the charge built on the electrode and has similar dependence on electrode potential, then the derivative of this layer cumulative charge would give the same dependence as the extracted DC. When the interfacial layer is thick enough (i.e., equal to one half of the length of electrolyte phase) then this condition is satisfied by definition. The question is how thin (or thick) the considered interfacial layer of electrolyte has to be in order to capture all trends observed in DC? In other words, we want to determine what region of EDL really matters for defining capacitance.

We have used two ways to calculate the distribution of charges in the z-direction. First, we associated the whole ion charge $\left(q_{c a l}=+1\right.$ or $\left.q_{a n}=-1\right)$ with the location of the ion center-of-mass and have utilized the density profiles of the ions' center-of-mass to compute the cumulative charge, i.e., 
$\sigma_{c o m}=\int_{0}^{\infty}\left(\rho_{+}(z) q_{c a t}+\rho_{-}(z) q_{a n}\right) d z$, where $\rho_{+}(\mathrm{z})$ and $\rho_{-}(\mathrm{z})$ are the ions' center-of-mass density profiles. In the second case, we used the distribution of partial atomic charges associated with each atom type to compute the cumulative charge, i.e., $\sigma_{\text {atom }}=\int_{0}^{d}\left(\sum_{i} \rho_{i,+}(z) q_{i, c a t}+\sum_{j} \rho_{j,-}(z) q_{j, a n}\right) d z$ where the summation is done over all atoms $i$ and $j$ belonging to cations and anions located within distance $d$ from the electrode surface and having a partial atomic charge $q_{i, i o n}$. Figure 6 shows the obtained charge derivatives for different layer widths calculated using both methods for the $\left[\mathrm{C}_{4} \mathrm{MIM}\right]\left[\mathrm{BF}_{4}\right]$ on $\mathrm{Au}(001)$. This figure shows that independently whether we use the center-of-mass or the atomic based calculations, the derivative of the charge accumulated in the interfacial layer of thickness 5.0 , which includes the first innermost layer of ions on the surface, gives quantitatively a very poor agreement with the true DC. This indicates that while some qualitative trends in DC might be captured by structural changes in the immediate (first) layer of ions at the surface, the outer layers clearly have an important contribution both in defining the dependence and the magnitude of DC as a function of electrode potential. Consideration of an interfacial layer of $\sim 8 \AA$ using the atom-based charge distributions seems to be enough to capture most of the features in DC. Yet, the same layer thickness for the canter-of-mass based charge distributions results in even qualitatively different dependence compared to $\mathrm{DC}$, therefore indicating that the orientation of ions and the corresponding distribution of partial atomic charges of the ions near the surface are quite important. However, as the layer thickness reaches $d \approx 12 \AA$ value, both descriptions result in quantitative agreement of electrolyte interfacial layer charge derivative with DC. Similar trends were observed for many other RTILs that have been investigated in our previous studies as illustrated in Figure 7. In this figure, we also show the contributions of individual ions to the derivative of the cumulative charge with interfacial layer of $d=10 \AA$ (i.e., contributions to DC) therefore allowing to clearly identify the rearrangement of which ions is more important at a given range of electrode potential. Based on the data presented in Figs. 6 and 7 as well as the results from simulation of other systems we can conclude that in typical RTILs the most important region of EDL, the one that is responsible for the charge storage in the capacitor, is only on the 
order of $1-2 \mathrm{~nm}$ wide. Electrolyte outside of this region is mostly screened out (from the electrode surface charges) by the formed EDL and basically represents a bulk, homogeneous electrolyte that does not participate in the charge storage process.

Finally, we would like to point out that similar magnitudes and dependencies of DC of various RTILs were obtained by MD simulations that have used the constant charge distribution approach for systems with atomically flat electrodes (mostly graphite or gold). This indicates that on the atomically flat surfaces while the instantaneous charge distribution can be quite broad (as shown in Figure 3a), on average the electrode surface atoms have very similar charge values and, therefore, for the sampling of EDL static properties the surface charge can be effectively approximated by a homogeneous charge distribution.

Influence of surface topography. While MD simulation predictions on atomically flat surfaces revealed important correlations between the EDL structure and DC as well as showed a good agreement with many experimental results, the source for the significant discrepancy with some other experimental data as well as the inconsistencies between experimental data themselves were not clear. A possible explanation of this issue has been provided by our recent MD simulations of RTIL electrolytes on atomically corrugated surfaces [xlviii,xlix]. In these simulations, we looked at the surfaces whose roughness patterns/features were comparable in size to the dimensions of ions comprising RTILs. Several such surfaces are shown in Figure 8. The simplest example of such a surface is the prismatic plane of graphite. The offset in layer stacking creates small groves on the surface with a width of $\sim 7 \AA$ and a depth of $1.4 \AA$ as illustrated in Figure 8. Using the same graphene sheets we have also created several more surfaces with different width and depth and have exposed them to several RTILs and specifically to $\left[\right.$ pyr $\left._{13}\right][\mathrm{FSI}]$ which is shown in Fig. 8. In the system labeled S3, the groves width is noticeably larger than the characteristic dimensions of both ions. Therefore, we refer to this surface as the one that has a roughness that is "weakly" correlated with electrolyte molecular dimensions. The surface S4, on the other hand, has surface pattern width that is similar to the ion dimensions, and therefore, we refer to this surface having the roughness with a "strong" correspondence to ions' dimensions. 
These simulations clearly demonstrated that slight differences in surface roughness can qualitatively change the dependence and the magnitude of DC (see Fig. 8) and can noticeably increase the energy storage of a capacitor. As we can see from Fig. 8 the systems with strong roughness show DC with multiple peaks in the same range of potentials where atomically flat surface showed very weak DC dependence. Even changing the basal plane graphite to prismatic plane results in a noticeable increase of DC magnitudes. What is particularly interesting is that for the system with strong correlation of roughness and ion dimensions, the DC magnitude can be a factor of four or five larger compared to that on the atomically flat surface. Similar effect we have observed in simulations of other RTILs indicating that this phenomenon is independent of chemical structure of the ions and primarily controlled by the correspondence of the ion dimensions and the size of the surface features. Strongly rough surfaces create steric effects that provide additional driving forces for specific orientation or arrangement of ions near the surface. This leads to very heterogeneous charge distribution on the electrode surface and the creation of large gradients in local electrostatic field, which, in turn, facilitates the co-ion and counter-ion rearrangement/separation. Effectively the heterogeneous polarization of the electrode by electrolyte magnifies the influence of the underlying surface topography. Due to rough electrostatic field profile near such surfaces the restructuring of EDL due to electrode potential change is not smooth. Instead it has several potential windows where the rearrangement is substantial therefore leading to sharp peaks in DC.

Our results obtained for atomically rough surfaces allowed us to suggest that the discrepancies observed in various experimental measurements shown in Fig. 1, as well as the deviations of simulation data presented in Figure 4 from some of those experiments, are likely due to unaccounted atomic roughness of the surface and/or restructuring of the electrode surfaces during experimental measurements. In dealing with realistic electrode surfaces, in practice, for many materials it might be difficult to have atomically flat surfaces because electrode surfaces in contact with ionic electrolyte can restructure and generate a topography that is different from the theoretical crystallographic orientation of interest. Therefore depending on the temperature and electrode/electrolyte materials combination, a roughening of an electrode surface in contact with electrolyte is possible [1,li]. For example, Su et al. [lii] indicated that 
$\mathrm{Au}$ (111) islands could form on top of the Au (001) electrode surfaces in contact with RTIL electrolyte. In situ experimental measurements on the Au (111) surface in contact with 1-butyl-1-methylpyrrolidinium tris(pentafluoroethyl)trifluorophosphate ([pyr $\left.\left.{ }_{14}\right][\mathrm{FAP}]\right)$ have identified a formation of alternating fcc/hcp surface structures upon reconstruction of cathodes [liii,liv] as well as a formation of potential-dependent islands in anodic region [lv]. Therefore, the results of our simulations shown in Figure 8 clearly demonstrate that experimental measurements of EDLCs have to pay a particular attention to characterization of the electrode surface structure.

Influence of ions chemical structure. Figure 4 has demonstrated that variations in the chemical structure of ions comprising RTIL electrolyte can make some differences to the shape of DC capacitance. However, on atomically flat surfaces these changes were not significant in magnitude. Then the obvious question is: does the dependence of DC on the ion chemical structure become more pronounced on atomically corrugated electrode surfaces? Taking into account our mentioned above arguments of the correspondence between the dimensions of the surface roughness and the ions, the intuitive answer to this question is yes. Clearly, since different ions have different shape and size they should differently respond to exposure to a charged electrode surface with a given surface roughness. Indeed, our simulations demonstrated that this is the case and Figure 9 illustrates this point for two series of RTILs in which anion was kept the same but alkyl tail length in the $C_{x}$ MIM cation was varied. Note that in Figure 9 instead of showing DC we plot the doubled value of the integral capacitance for the whole capacitor $\left(\mathrm{IC}_{\text {cap }}\right)$ as a function of applied potential difference. Many experimental data are reported this way and we adapted similar representation. Since the total capacitance of the capacitor is related to the capacitance on individual electrodes as $1 / \mathrm{IC}_{\text {cap }}=1 / \mathrm{IC}_{+}+\mathrm{i} / \mathrm{IC}$, the multiplication of $\mathrm{IC}_{\text {cap }}$ by two is just for convenience of comparison with magnitudes of ICs of individual electrodes. Finally, the IC vs applied potential usually looks smoother compared to DC vs electrode potentials because IC represents an average (integrated) DC over a certain potential window.

In Figure 9 we only compare the basal and prismatic plane graphite surfaces. Nevertheless, we can clearly see that for the systems with FSI anion even small changes in surface roughness result in a 
noticeable change of the capacitance, particularly at relatively low potential differences. The prismatic graphite surface generates almost doubled capacitance on the basal plane for RTILs with small alkyl tails ( $n=2$ and 4). As the applied potential difference increases or the alkyl tail length increases the difference in the capacitances generated by prismatic and basal plane surfaces is decreasing. Interestingly, we found that a replacement of the FSI anion with a larger TFSI anion reduces the difference between capacitances obtained on flat and corrugated electrode surfaces as well as it reduces the effect of the cation tail length. Therefore, on the atomically corrugated surface the choice of cation/anion combination should play a more significant role in the EDL structure and generated capacitances compared to those on atomically flat electrodes.

Temperature dependence. Finally, we examine the influence of temperature on the EDL structure and generated capacitances. Figure 10 summarizes these results based on MD simulations of [pyr $\left.{ }_{13}\right]$ [FSI] RTIL on graphite surfaces of different roughness (systems S1-S4 shown in Figure 8) and various temperatures. On atomically flat and weakly rough surfaces, we found a decrease of DC and IC versus temperature within a typical potential window of interest where RTILs are stable (i.e. within $-2 \mathrm{~V}$ to $+2 \mathrm{~V}$ window). This was associated with an increasing entropic penalty of the ordered EDL structure as the temperature increases. In other words, with increasing temperature the interfacial layer(s) become more disordered and have a lower density of counterions. This leads to the reduction of charge separation between coions and counterions in the EDL and, therefore, lowers the capacitance. The predicted from our simulations weak dependence of DC vs. temperature for the flat and weakly rough surfaces is in excellent agreement with experimental work of Druschler et al. [xxxvii] and the analytical theories such as GouyChapman [lvi] and Kornyshev [xxviii] models.

On atomically rough surfaces, where the dimensions of surface patters are comparable to ion dimensions, a much more pronounced oscillation in DC and much stronger temperature dependence is observed, particularly at low voltages. However, at larger voltages the temperature dependence of DC becomes weak and shows similar trends as on flat surfaces. As a general trend, on atomically rough 
surface the increase of temperature flattens the overall shape of DC vs potential, i.e., the oscillations between minima and maxima in DC become smaller at higher temperatures. The observed weak temperature dependence of capacitance for the flat and weakly rough surfaces is consistent with large electrostatic interactions between ions near electrode surface [lvii] and even substantial temperature variations (from $363 \mathrm{~K}$ to $533 \mathrm{~K}$ ) do not provide sufficient thermal energy to dramatically change the EDL composition (characterized by the density profiles not shown here). For the strongly rough surfaces, the large electrostatic fields generated near the rough surface edges combined with the steric effects provide additional driving forces for the charge separation and EDL restructuring that can noticeably change within a small temperature interval. While the DC shape for the strongly rough surface changes dramatically (qualitative) upon temperature increase from $363 \mathrm{~K}$ to $463 \mathrm{~K}$, the changes in DC upon increasing temperature from $463 \mathrm{~K}$ to $533 \mathrm{~K}$ are rather small. This suggests that the strongly sensitive (to electrode potential) DC at lower temperature (363K) and the disappearance of this sensitivity at higher temperatures are likely related to the steric constrains of electrolyte ions that are partially inserted into atomic scale patterns on the surface.

Finally, there are some experimental works, including very recent ones [xxxvi], that showed a systematic and large increase of DC with increasing temperature in the entire electrode potential range investigated, including relatively large voltages. For example, from the data reported in Ref. [v] for $\left[\mathrm{C}_{4} \mathrm{MIM}\right]\left[\mathrm{PF}_{6}\right]$ on $\mathrm{Pt}$ it follows that $\mathrm{DC}$ and IC at $+1.5 \mathrm{~V}$ are about factor of $2-2.5$ larger at $70^{\circ} \mathrm{C}$ than at $20^{\circ} \mathrm{C}$, which means that with increasing temperature the rate of counterion-coion imbalance near electrode surfaces increases by a factor of 2-2.5. Our simulations show that the temperature does not change the density of the interfacial layer significantly and therefore cannot promote a significantly more ion separation at higher temperatures than at low temperatures. Moreover, we find that the charge is already mostly separated at these high voltages (independently of the surface structure) and hence again, it is hard to envision how any perturbations on the system can result in substantial changes in the EDL structure that can lead to a factor of two increase of the rate of charge separation (i.e., capacitance). Our simulations 
essentially rule out the experimentally reported observations of systematic (and large) increase of the nonFaradic capacitance of RTILs with increasing temperature. We believe that those experiments should be re-examined in light of our simulations [lviii] and the recent experimental analysis of Druschler et al. [xxxvii] which reported additional slow relaxation mechanism(s) that is not associated with electrolyte EDL restructuring but has a strong temperature dependence [xxix].

\section{Conclusions}

In summary, we would like to emphasize that MD simulations of RTILs on charged electrode surfaces can provide an important molecular level understanding of the structure and correlations in the electric double layer formed by electrolyte at electrode surfaces as well as help to understand the charge storage mechanisms in EDL capacitors. Taking into account that in these systems the charged is stored in a very thin, 1-2nm wide interfacial layers, utilization of atomistic, chemically realistic models in such simulations is critical. Details of atomic partial charge distributions in electrolyte molecules, ions orientation, distribution of induced charges on the electrode surface, and other atomic scale details can play a significant role in defining the storage capacitance. We demonstrated that on atomically flat surfaces, a large number of different RTILs show very similar values for capacitance with a very weak dependence on the electrode potential. In contrast, the same RTILs on atomically corrugated surfaces, where the surface roughness features are comparable with dimensions of the ions, the capacitance magnitude as well as its dependence on the electrode potential can be significant and complex. These results provide a possible explanation why many of the previous experimental measurements of very similar RTIL/electrode combinations were producing qualitatively and quantitatively different results. We believe that any experimental data on the capacitance of RTIL-based electrolytes must be considered taking into account the details of the atomic scale topography of the electrode surface. While the atomic scale surface structure can significantly complicate the analysis and comparison of experimental data, at the same time our simulations show that overall it promotes the charge separation in the electrolyte electric double layer and hence, can noticeably increase the energy storage capacitance. This indicates that 
significant improvements in capacitive energy storage can be achieved by optimizing the atomistic scale correlations between electrolyte chemical and electrode surface structures.

\section{Acknowledgement.}

The authors are grateful to the Department of Energy for financial support under the grant DESC0001912 to the University of Utah and the grant DE-AC02-05CH11231 on PO No. 6838611 to the University of Utah. 


\section{References}

[i] Conway, B.E. Electrochemical Supercapacitors: Scientific Fundamentals and Technological Applications. Kluwer, (1999).

[ii] P. Simon, Y. Gogotsi, Nature Materials 7 (2008) 845.

[iii] Y. Z. Su, Y.C. Fu, J.W. Yan, Z.B. Chen, B.W. Mao, Angew. Chem. Int. Ed. 48 (2009) 5148.

[iv] M.T. Alam, M. Md. Islam, T. Okajima, T. Ohsak, Electrochem. Commun. 9 (2007) 2370.

[v] F. Silva, C. Gomes, M. Figueiredo,R. Costa, A. Martins, C.M. Pereira, J. Electroanal. Chem. 622, (2008) 153.

[vi] V. Lockett, M. Horne, R. Sedev, T. Rodopoulos, J. Ralston, Phys. Chem. Chem. Phys. 12 (2010) 12499.

[vii] S. Baldelli, Acc. Chem. Res., 41 (2008) 421.

[viii] J. Chmiola, G. Yushin, Y. Gogotsi, C. Portet, P. Simon, P. Taberna, Science 313 (2006) 1760.

[ix] T.A. Centeno, O. Sereda, F. Stoeckli, Phys. Chem. Chem. Phys. 13 (2011) 12403.

[x] J. Vatamanu, Z. Hu, D. Bedrov, C. Perez, Y. Gogotsi, J. Phys. Chem. Lett. 4 (2013) 2829.

[xi] L.B. Bhuiyan, S. Lamperski, J. Wu, D. Henderson, J. Phys. Chem. B 116 (2012) 10364.

[xii] L.B. Bhuiyan, D. Henderson, S. Sokolowski, Condensed Matter Physics 15 (2012) 23801.

[xiii] L.B. Bhuiyan, C.W. Outhwaite, D. Henderson, J. Chem. and Eng. Data 56 (2011) 4556.

[xiv] L.B. Bhuiyan, D. Henderson, Mol. Phys. 109 (2011) 1863.

[xv] L.B. Bhuiyan, D. Henderson, Mol. Simulation. 37 (2011) 269.

[xvi] C.W. Outhwaite, S. Lamperski, L.B. Bhuiyan, Molecular Physics 109 (2011) 21.

[xvii] W. Silvestre-Alcantara, L.B. Bhuiyan, D. Henderson J. Chem. and Eng. Data 55 (2010) 1837.

[xviii] W. Silvestre-Alcantara, L.B. Bhuiyan, C.W. Outhwaite, D. Henderson, Collection of Czechoslovak Chemical Comm. 75 (2010) 425.

[xix] D. Henderson, L.B. Bhuiyan, J. Chem. Theory and Comput. 5 (2009) 1985.

[xx] D. Henderson, S. Lamperski, Z. Jin, J. Wu, J. Phys. Chem. B 115 (2011) 12911.

[xxi] M. Marinescu, M. Urbakh, A.A. Kornyshev, Phys. Chem. Chem. Phys. 14 (2012) 1371.

[xxii] A.A. Kornyshev, M. Marinescu, J. Paget, M. Urbakh, Phys. Chem. Chem. Phys. 14 (2012) 1850. 
[xxiii] M.Z. Bazant, B.D. Storey, A.A. Kornyshev, Phys. Rev. Lett. 106 (2011) 046102.

[xxiv] Z.Y. Wang, Y.Q. Ma, Phys. Rev. E. 85 (2012) 062501.

[xxv] T. Nagy, D. Henderson, D. Boda, J. Phys. Chem. B 115 (2011) 11409.

[xxvi] S. Lamperski, C.W. Outhwaite, L.B. Bhuiyan, J. Phys. Chem. B 113 (2009) 8925.

[xxvii] L. Kenji, S. Hirofumi, J. Chem. Phys. 135 (2011) 244702.

[xxviii] A.A. Kornyshev, J. Phys. Chem. B 111 (2007) 5545.

[xxix] B. Roling, M. Druschler, B. Huber, Faraday Discuss. 154 (2012) 303.

[xxx] M. Druschler, B. Huber, B. Roling, J. Phys. Chem. C 115 (2011) 6802.

[xxxi] V. Lockett, R. Sedev, J. Ralston, M. Horne, T. Rodopoulos, J. Phys. Chem. C, 112 (2008) 7486.

[xxxii] R. Costa, C M. Pereira, F. Silva, Phys. Chem. Chem. Phys., 12 (2010) 11125.

[xxxiii] M. Md. Islam, M. T. Alam, T. Ohsaka, J. Phys. Chem. C, 112 (2008) 16568.

[xxxiv] M. T. Alam, J. Masud, M. Md. Islam, T. Okajima, T. Ohsaka, J. Phys. Chem. C, 115 (2011) 19797.

[xxxv] M. T. Alam, M. Md. Islam, T. Okajima, T. Ohsaka, J. Phys. Chem. C, 112 (2008) 16600.

[xxxvi] L. Siinor, R. Arendi, K. Lust, E. Lust, J. Electroanal. Chem., 689 (2013) 51.

[xxxvii] M. Druschler, N. Borisenko, J. Wallauer, C. Winter, B. Huber, F. Endresb, B. Roling, Phys. Chem. Chem. Phys. 2012, 14, 5090- 5099.

[xxxviii] M. T. Alam, M. Md. Islam, T. Okajima, T. Ohsaka, J. Phys. Chem. C, 2007, 111, 18326-18333.

[xxxix] C. Cannes, H. Cachet, C. Debiemme-Chouvy, C. Deslouis, J. de Sanoit, C. Le Naour, V. A. Zinovyeva, J. Phys. Chem. C, 117 (2013) 22915.

[xl] J. Vatamanu, O. Borodin, G.D. Smith, Phys. Chem. Chem. Phys. 12 (2010) 170.

[xli] S.K. Reed, O.J. Lanning, P.A. Madden, J. Chem. Phys. 126 (2007) 084704.

[xlii] J.I. Siepmann, M. Sprik, J. Chem. Phys. 102 (1995) 511.

[xliii] L. Dai, Acc. Chem. Res. 46 (2013) 31.

[xliv] C. Merlet, C. Pean, B. Rotenberg, P.A. Madden, P. Simon, M. Salanne J. Phys. Chem. Lett. 4 (2013) 4, 264. 
[xlv] A.A. Kornyshev, N.B. Luque, W. Schmickler J. Solid State Electrochem, 18 (2014) 1345.

[xlvi] S. Lamperski, C.W. Outhwaite, L.B. Bhuiyan, J. Phys. Chem. B 113 (2009) 6569.

[xlvii] H. Gerischer, R. McIntyre, D. Scherson, W. Storck J. Phys. Chem. 91 (1987) 1930.

[xlviii] L. Xing, J. Vatamanu, G.D. Smith, D. Bedrov, J. Phys. Chem. Lett. 3 (2012) 1124.

[xlix] J. Vatamanu, L. Cao, O. Borodin, D. Bedrov, G.D. Smith, J. Phys. Chem. Lett., 2 (2011) 2267.

[1] R.T. Gore, T. Bond, W. Zhang, R.W.J. Scott, I.J. Burgess, Electrochem. Commun. 12 (2010) 1340.

[li] Y. Su, J. Yan, M. Li, M. Zhang, B. Mao, J. Phys. Chem. C, 117 (2013) 205.

[lii] Y.-Z. Su, Y.-C. Fu, J.-W. Yan, Z.-B. Chen, B.-W. Mao, Angewandte Chemie, International Edition, 48 (2009) 5148.

[liii] R. Atkin, N. Borisenko, M. Druschler, S. Z. El. Abedin, F. Endres, R. Hayes, B. Huber and B. Roling, Phys. Chem. Chem. Phys., 13 (2011) 6849.

[liv] R. Hayes, N. Borisenko, M. K. Tam, P. C. Howlett, F. Endres, R. Atkin, J. Phys. Chem. C, 115 (2011) 6855.

[lv] F. Endres, N. Borisenko, S. Z. El Abedin, R. Hayes and R. Atkin, Faraday Discuss. 154 (2012) 221. [lvi] (a) G. Gouy, Compt. Rend. 149 (1910) 654. (b) D.L. Chapman, Philos. Mag. 25 (1913) 475.

[lvii] O. Borodin, W. Gorecki, G. D. Smith, M. Armand, J. Phys. Chem. B., 114 (2010) 6786. [lviii] J. Vatamanu, L. Xing, W. Li, D. Bedrov, Phys. Chem. Chem. Phys, 16 (2014) 5174. 


\section{Figure Captions.}

Figure 1. Compilation of experimental data of differential capacitance measured on similar RTILs on gold, platinum, and glassy carbon electrodes.

Figure 2. Schematic illustration of simulation cell set up, typical charge density distribution (top panel) and the Poisson potential (bottom panel) in the direction perpendicular to electrode surfaces as obtained from MD simulations using the constant applied potential approach.

Figure 3. Distribution of the induced charges on electrode surface atoms as obtained from simulations using the constant applied potential for two RTILs on atomically flat basal plane graphite (top) and atomically corrugated prismatic graphite (bottom).

Figure 4. Differential capacitance as a function of electrode potential for several RTILs at the basal plane graphite and $\mathrm{Au}(001)$ atomically flat electrode surfaces at $363 \mathrm{~K}$ as obtained from atomistic MD simulations using the constant potential approach.

Figure 5. Atom density profiles for various groups of $\left[\mathrm{C}_{4} \mathrm{MIM}\right]\left[\mathrm{PF}_{6}\right]$ RTIL on graphite and $\mathrm{Au}$ surfaces: (a) anion fluorine (b) $\mathrm{C}_{4} \mathrm{MIM}$ ring carbon atoms, (c) carbon atoms from the $\mathrm{C}_{4} \mathrm{MIM}$ alkyl tail.

Figure 6. Differential capacitance and the derivatives of the $\left[\mathrm{C}_{4} \mathrm{MIM}\right]\left[\mathrm{PF}_{6}\right]$ electrolyte charge density located within interfacial layers of various width $d$ near the electrode surface. For convenience of comparison the derivatives of electrolyte charge densities were multiplied by -1 and rescaled into units of $\mu \mathrm{F} / \mathrm{cm}^{2}$. The charge densities were computed based on (a) the locations of ions center-of-mass and (b) the locations of and the corresponding partial charges.

Figure 7. Differential capacitances and the derivatives of cumulative charge density (converted to $\mu \mathrm{F} / \mathrm{cm}^{2}$ units and multiplied by -1 for convenience of comparison) of electrolyte located within the interfacial

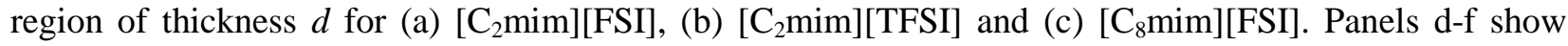
contributions of anions and cations to the derivatives of the total charge within the interfacial layer of $d=10$.

Figure 8. Differential capacitance as a function of applied potential for atomically flat and rough graphite electrode surfaces in contact with $\left[\mathrm{Pyr}_{13}\right][\mathrm{FSI}] \mathrm{RTIL}$ as obtained from atomistic MD simulations.

Figure 9. Doubled integral capacitance of the entire capacitor $\left(2 * \mathrm{IC}_{\text {cap }}\right)$ as a function of potential difference between electrodes for (a) $\left[\mathrm{C}_{\mathrm{n}} \mathrm{mim}\right][\mathrm{FSI}]$ and (b) $\left[\mathrm{C}_{\mathrm{n}} \mathrm{mim}\right][\mathrm{TFSI}]$ ionic liquids on atomically flat basal plane graphite (open symbols) and corrugated prismatic plane graphite (filled symbols) electrode surfaces.

Figure 10. a) Differential capacitance as obtained at several temperatures on electrode surfaces with different surface topography in contact with [Pyr $\left.{ }_{13}\right]$ [FSI] RTIL. b) Temperature dependence of the integral capacitance for different electrode surfaces. 
Figure 1.
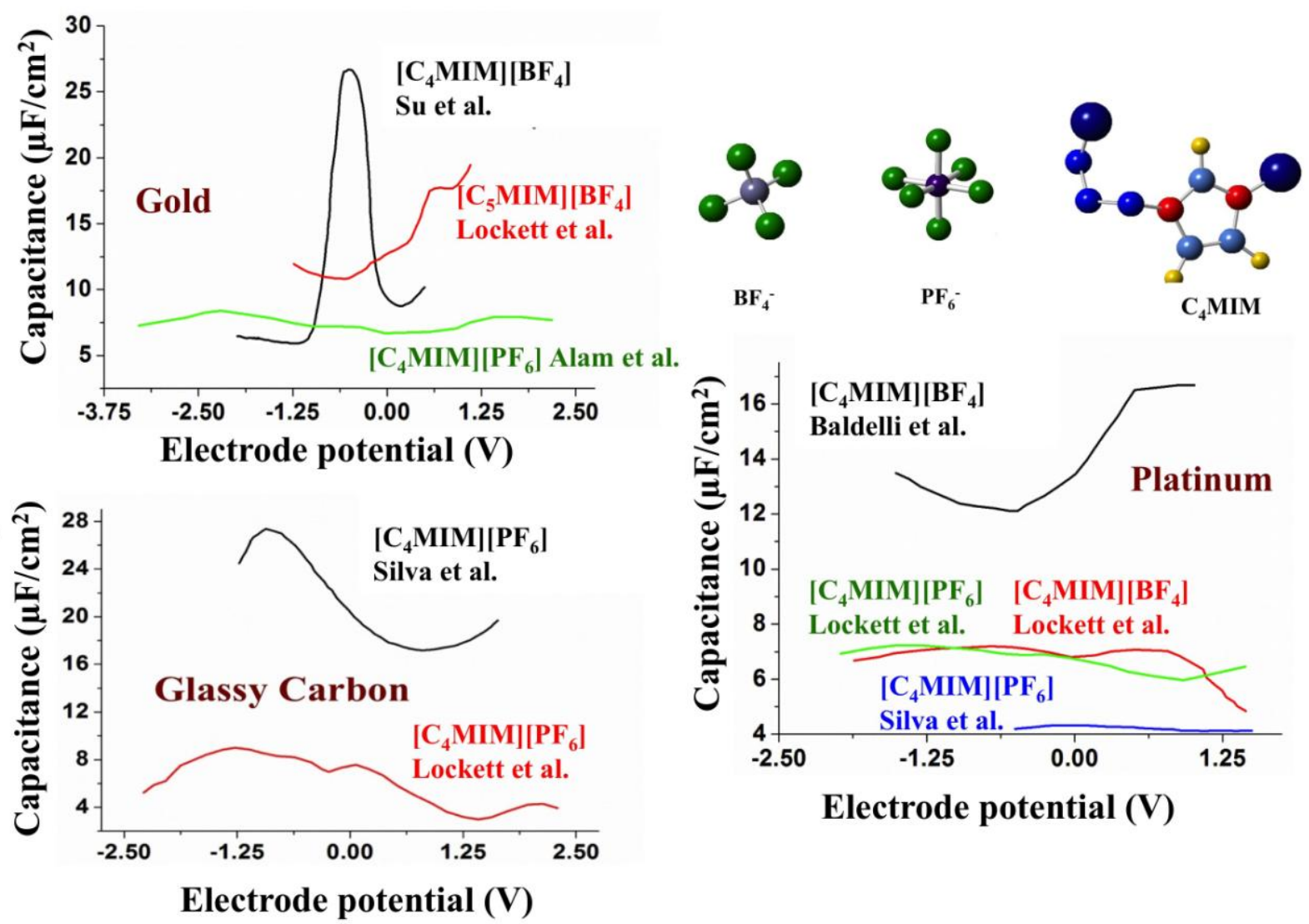

Figure 1. A compilation of experimental data of differential capacitance measured on similar RTILs on gold, platinum, and glassy carbon electrodes. 
Figure 2.
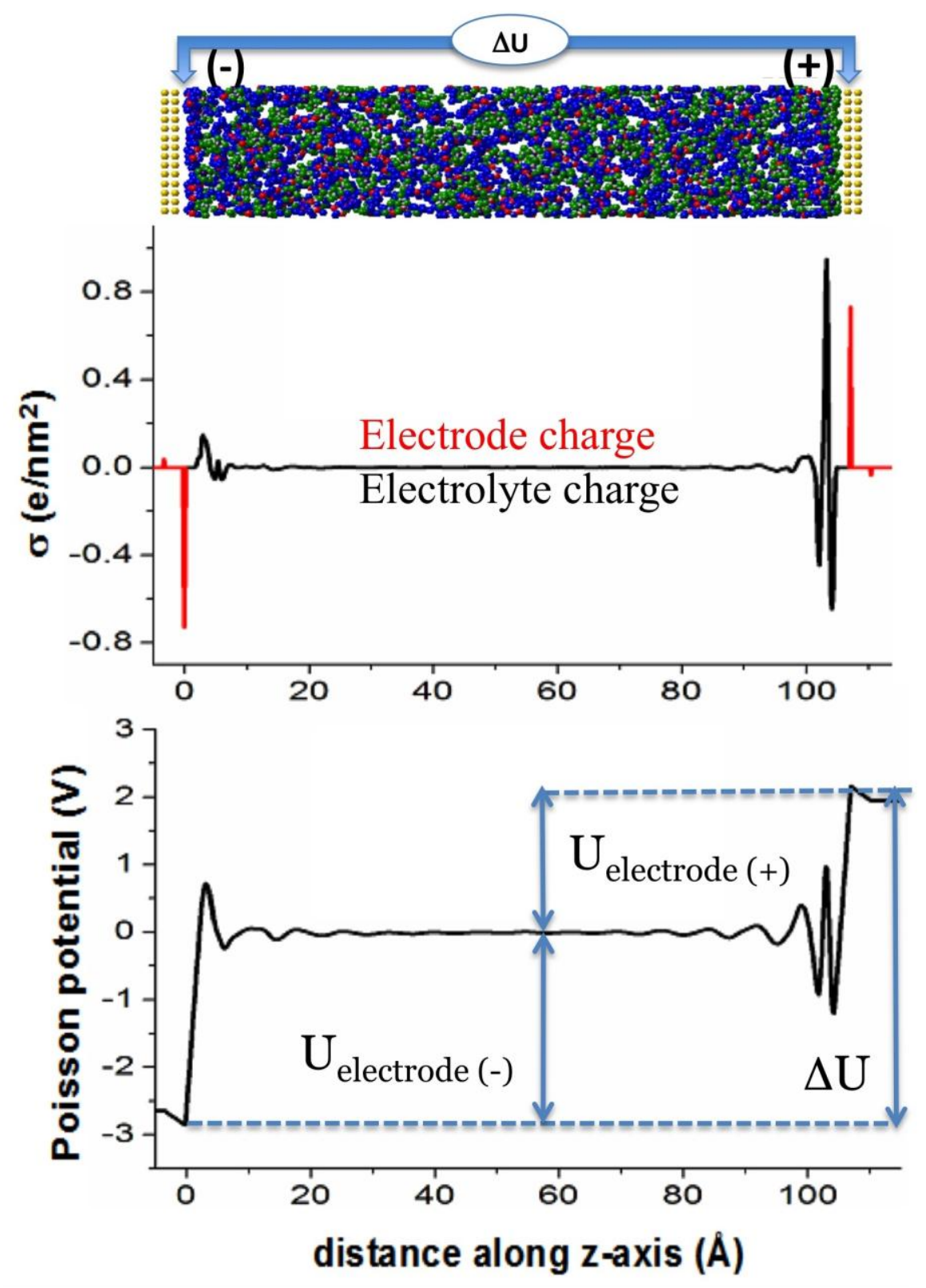

Figure 2. A schematic illustration of simulation cell set up, typical charge density distribution (top panel) and the Poisson potential (bottom panel) in the direction perpendicular to electrode surfaces as obtained from MD simulations using the constant applied potential approach. 
Figure 3

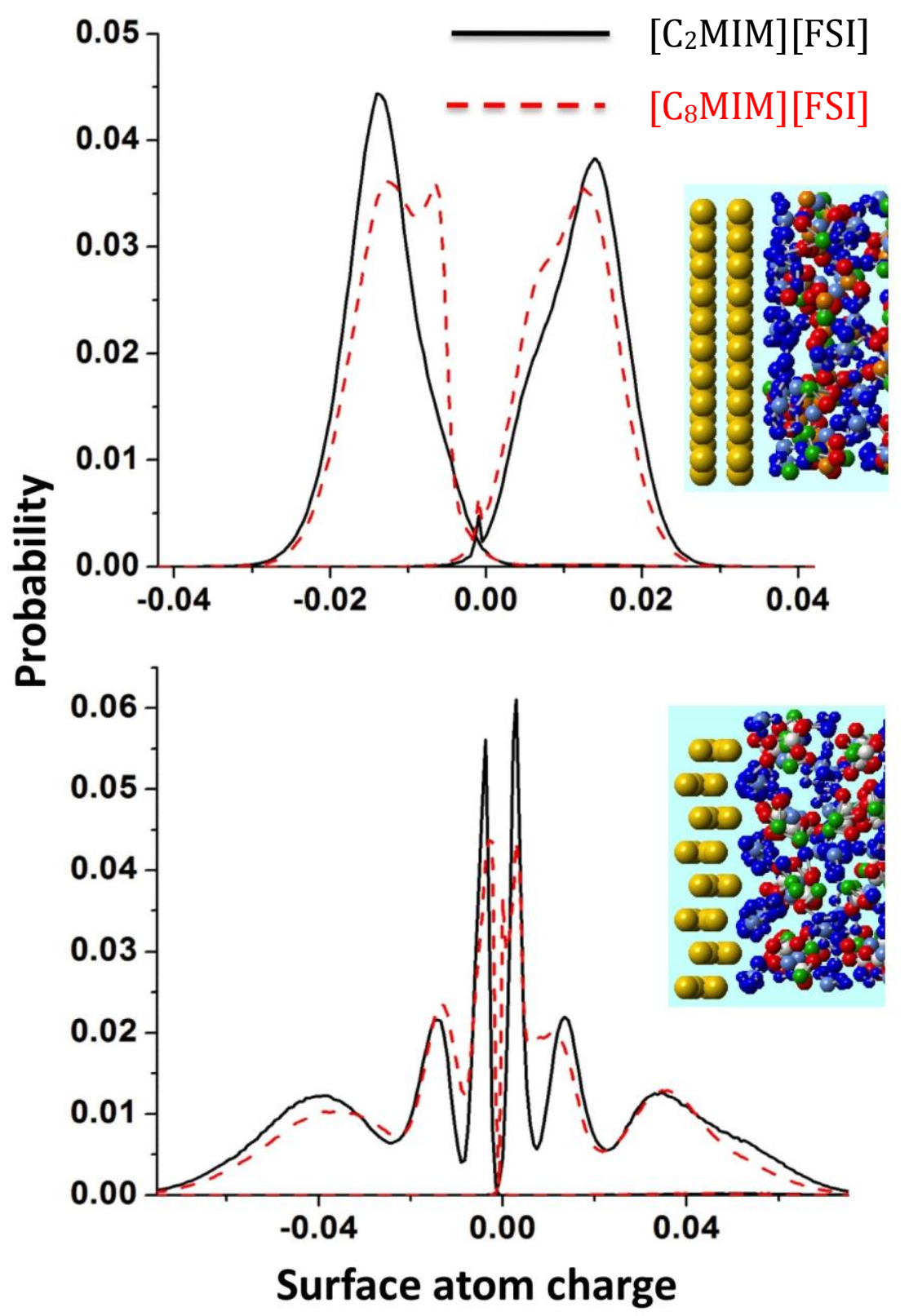

Figure 3. The distribution of the induced charges on electrode surface atoms as obtained from simulations using the constant applied potential for two RTILs on atomically flat basal plane graphite (top) and atomically corrugated prismatic graphite (bottom). 
Figure 4.

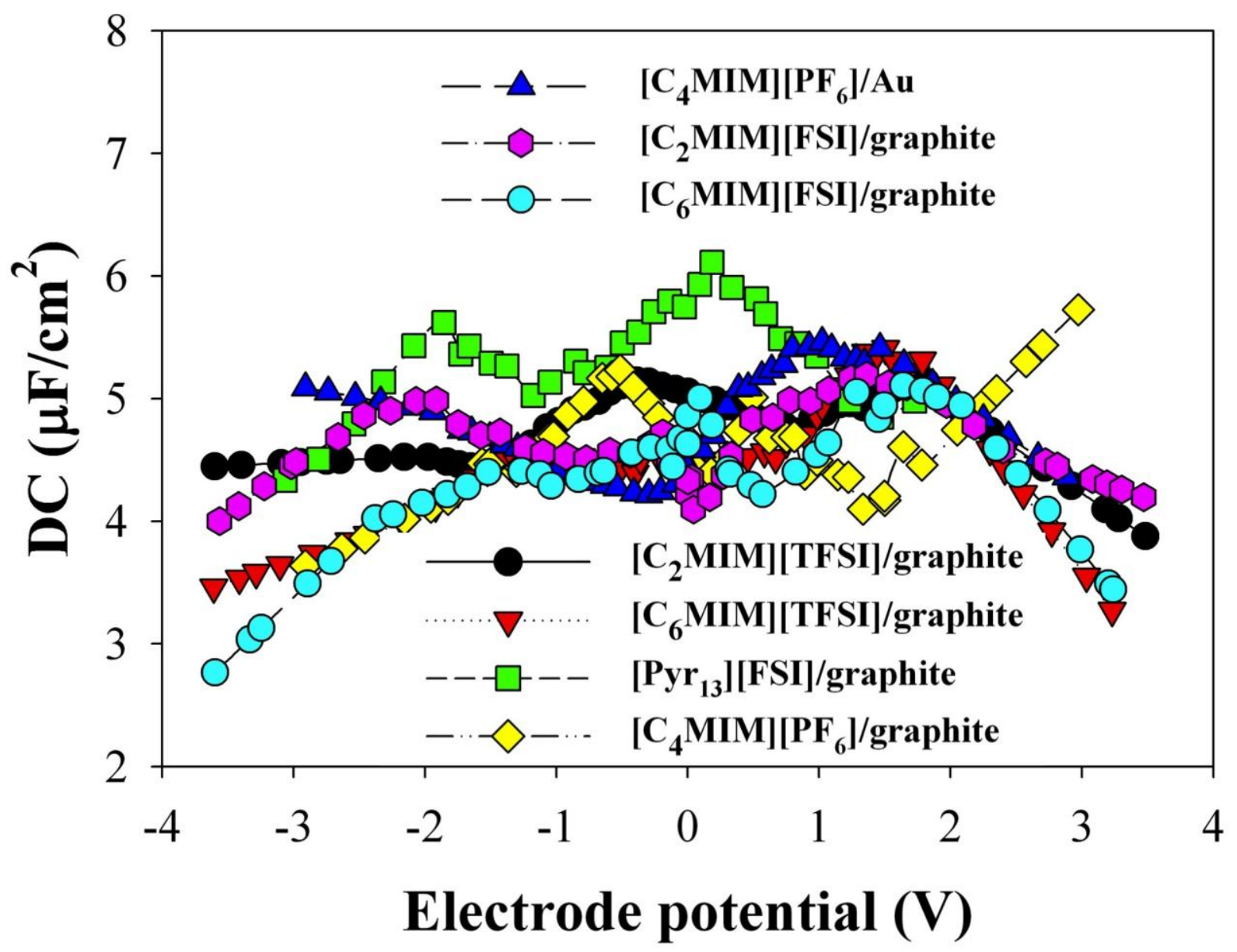

Figure 4. The differential capacitance as a function of electrode potential for several RTILs at the basal plane graphite and $\mathrm{Au}(001)$ atomically flat electrode surfaces at $363 \mathrm{~K}$ as obtained from atomistic MD simulations using the constant potential approach. 
Figure 5.

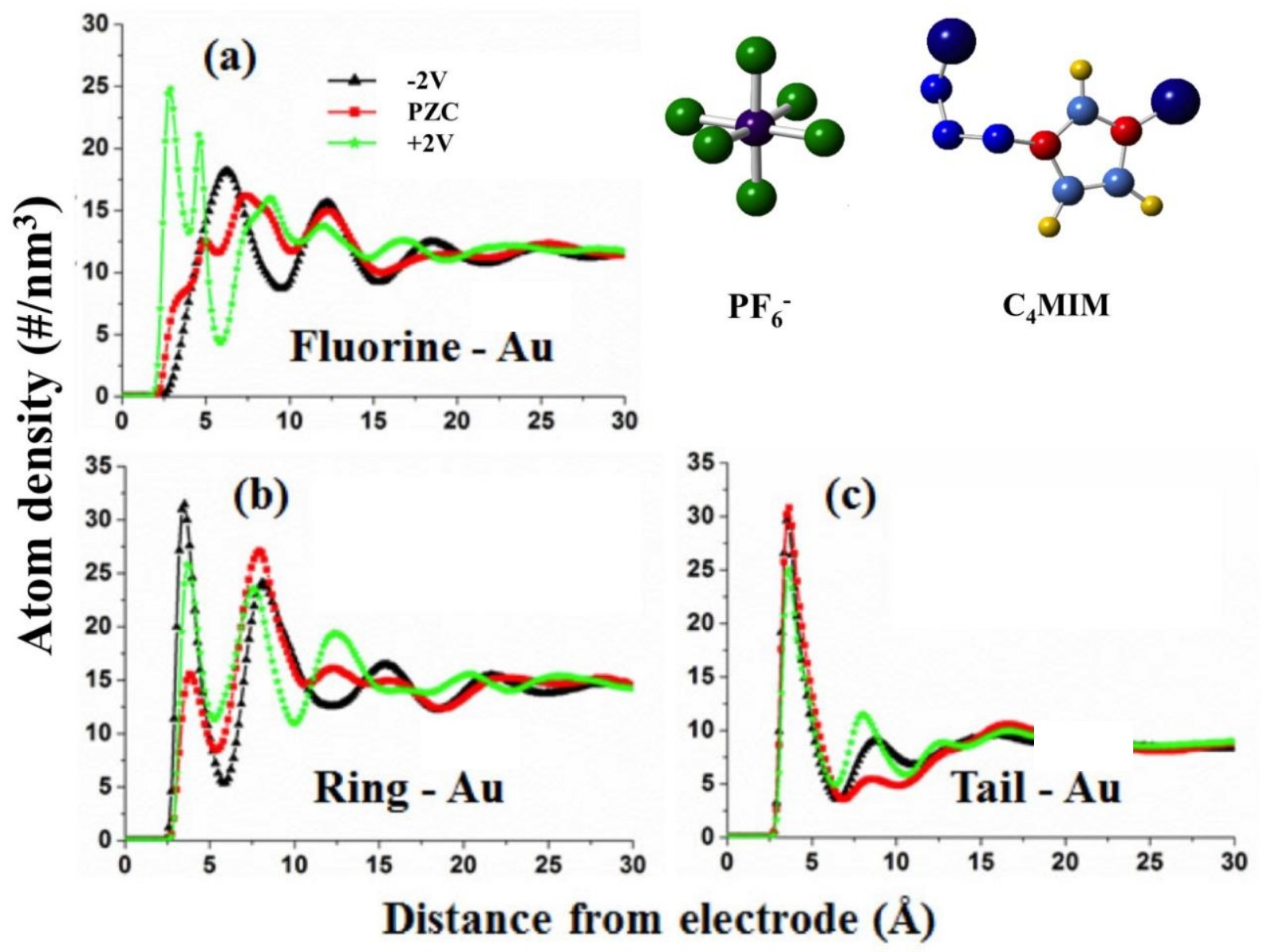

Figure 5. The atom density profiles for various groups of [ $\left.C_{4} M I M\right]\left[P F_{6}\right] R T I L$ on graphite and $A u$ surfaces: (a) anion fluorine (b) $C_{4} M I M$ ring carbon atoms, (c) carbon atoms from the $C_{4} M I M$ alkyl tail. 
Figure 6
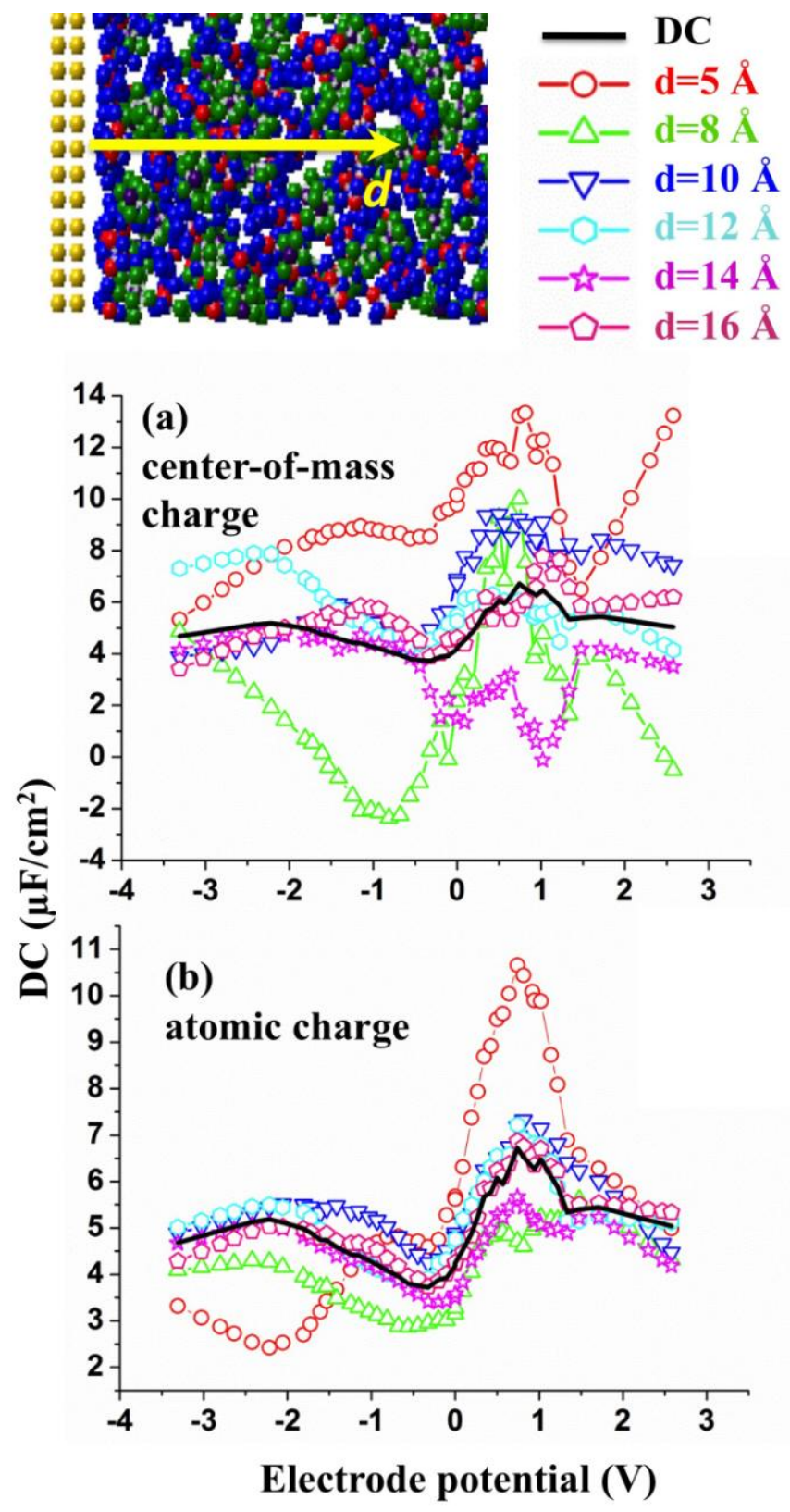

Figure 6. The differential capacitance and the derivatives of the $\left[C_{4} M I M\right]\left[P F_{6}\right]$ electrolyte charge density located within interfacial layers of various width $d$ near the electrode surface. For convenience of comparison the derivatives of electrolyte charge densities were multiplied by -1 and rescaled into units of $\mu F / \mathrm{cm}^{2}$. The charge densities were computed based on (a) the locations of ions center-of-mass and $(b)$ the locations of and their corresponding partial charges. 
Figure 7
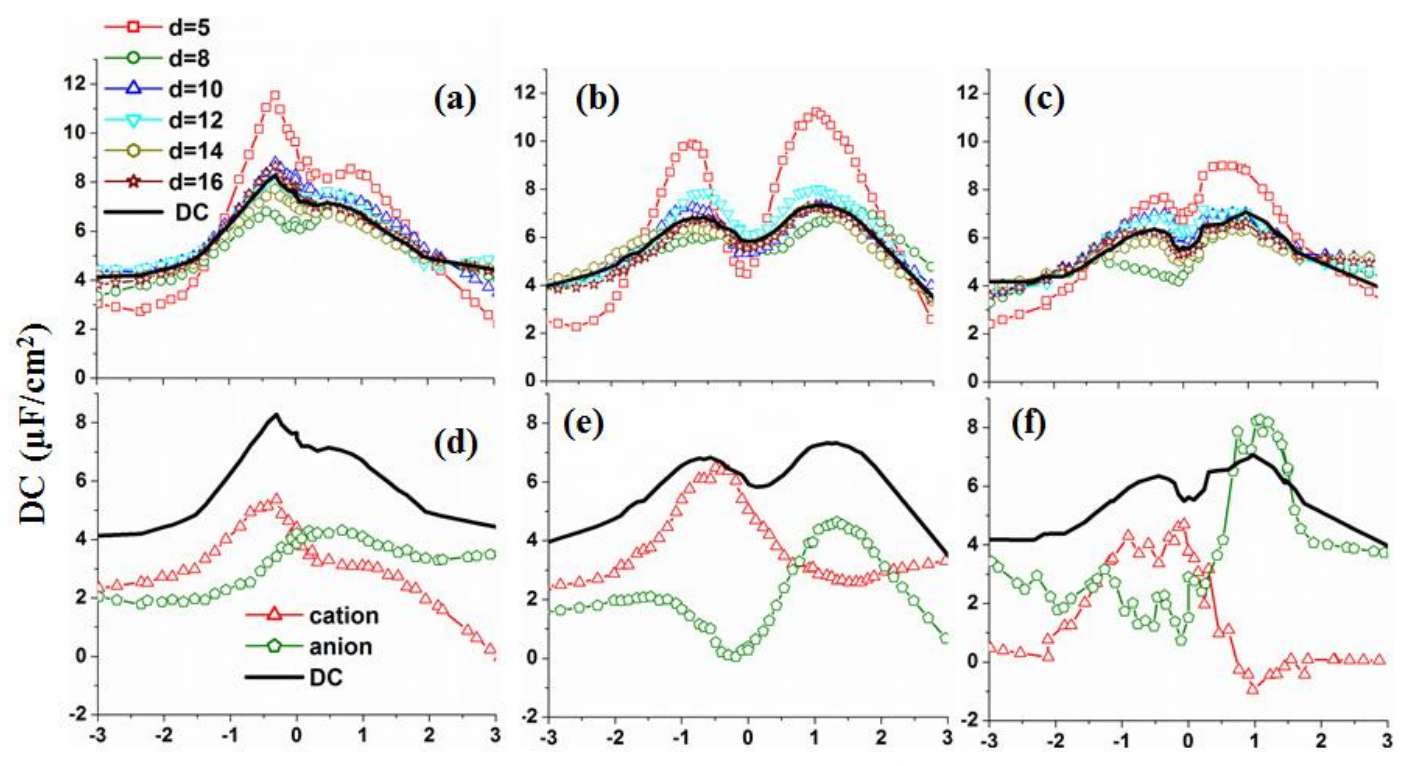

Electrode potential (V)

Figure 7. The differential capacitances and the derivatives of cumulative charge density (converted in $\mu \mathrm{F} / \mathrm{cm}^{2}$ and multiplied by -1 for convenience of comparison) of electrolyte located within the interfacial region of thickness $d$ for (a) [ $C_{2}$ mim] [FSI], (b) [ $C_{2}$ mim] [TFSI] and (c) [ $C_{8}$ mim] [FSI]. The panels d-f show contributions of anions and cations to the derivatives of the total charge within the interfacial layer of $d=10$. 
Figure 8

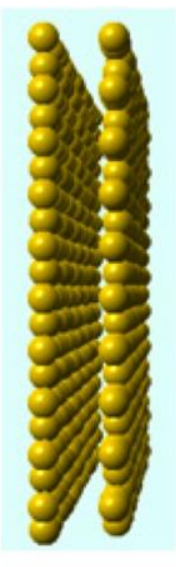

(S1)

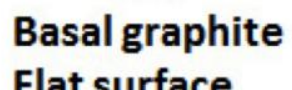

Flat surface

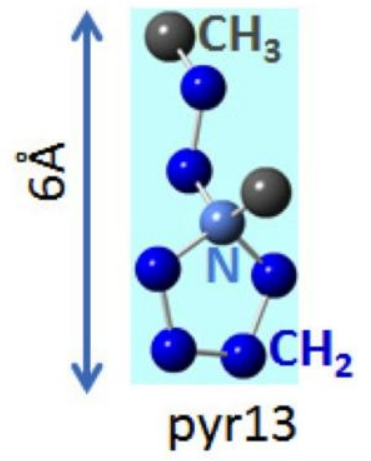

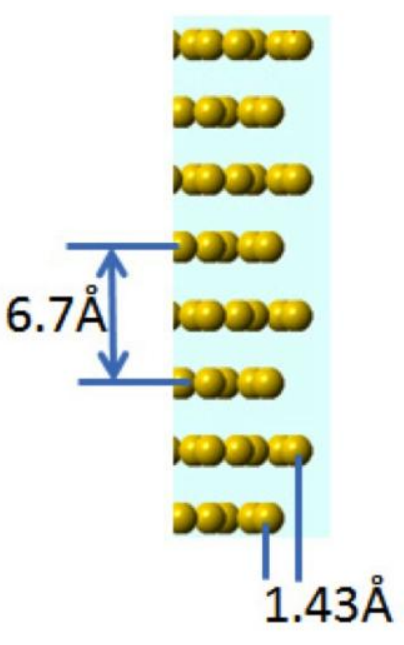

(S2)

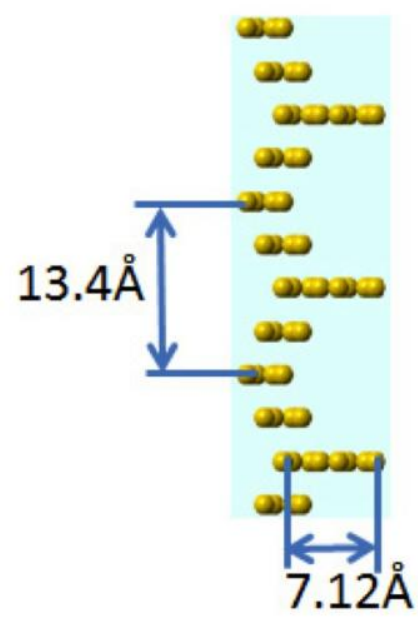

(S3)

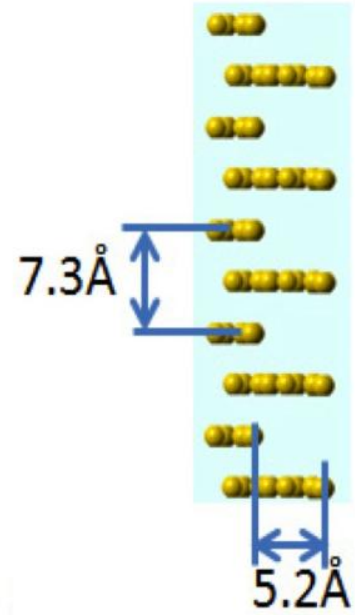

(S4)

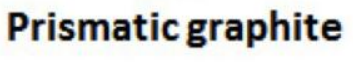

Weak roughness

Strong roughness

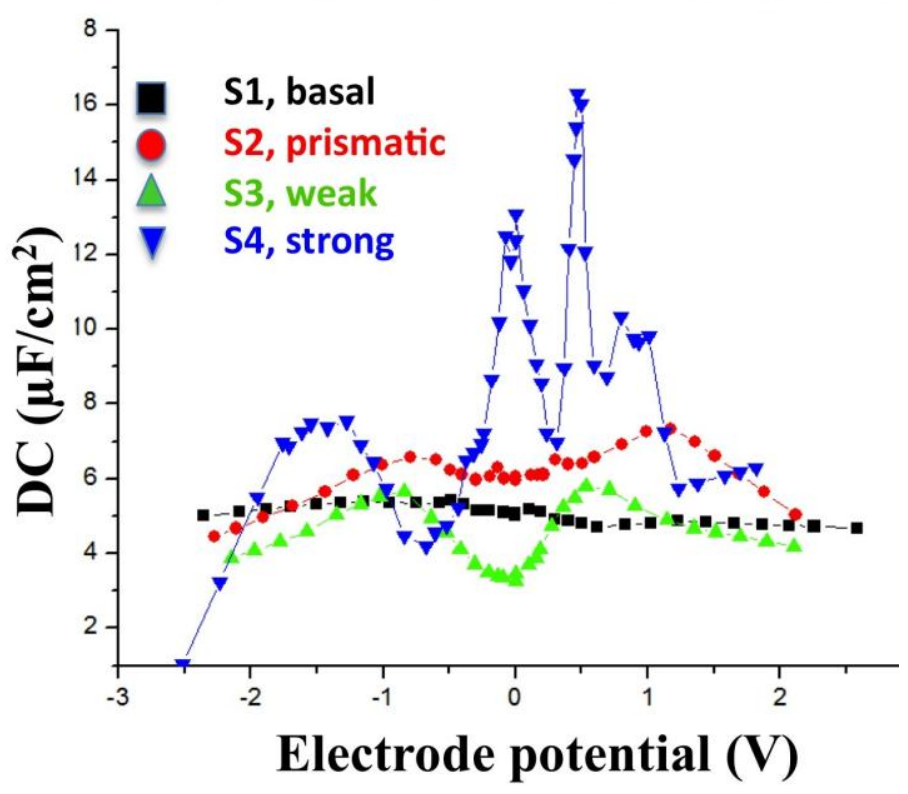

Figure 8. The differential capacitance as a function of applied potential for atomically flat and rough graphite electrode surfaces in contact with [Pyr $\left.{ }_{13}\right][F S I]$ RTIL as obtained from atomistic MD simulations. 
Figure 9.
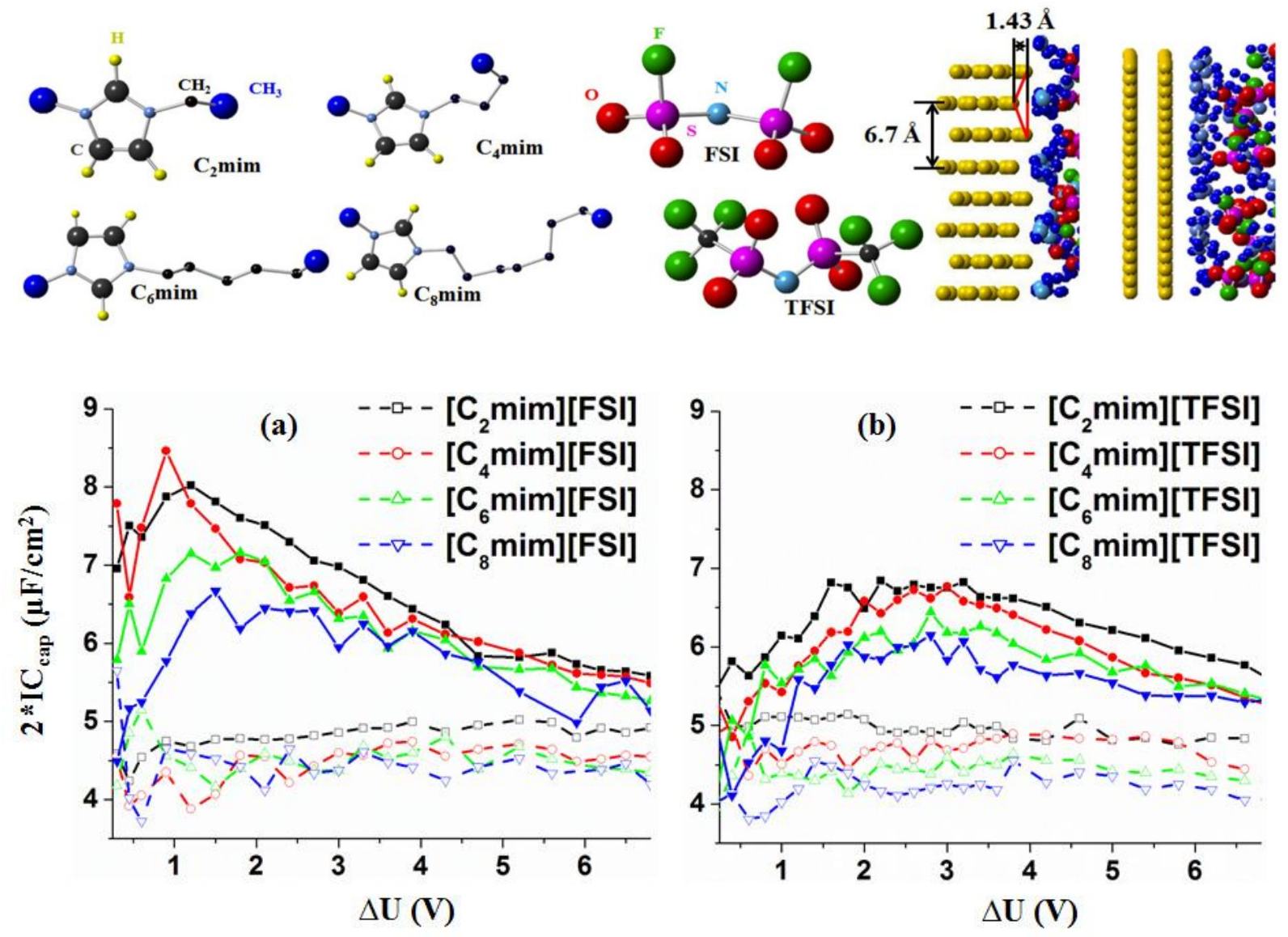

Figure 9. The doubled integral capacitance of the entire capacitor $\left(2 * I C_{\text {cap }}\right)$ as a function of potential difference between electrodes for (a) $\left[C_{n} \mathrm{mim}\right][\mathrm{FSI}]$ and (b) $\left[C_{n} \mathrm{mim}\right][\mathrm{TFSI}]$ ionic liquids on atomically flat basal plane graphite (open symbols) and corrugated prismatic plane graphite (filled symbols) electrode surfaces. 
Figure 10.

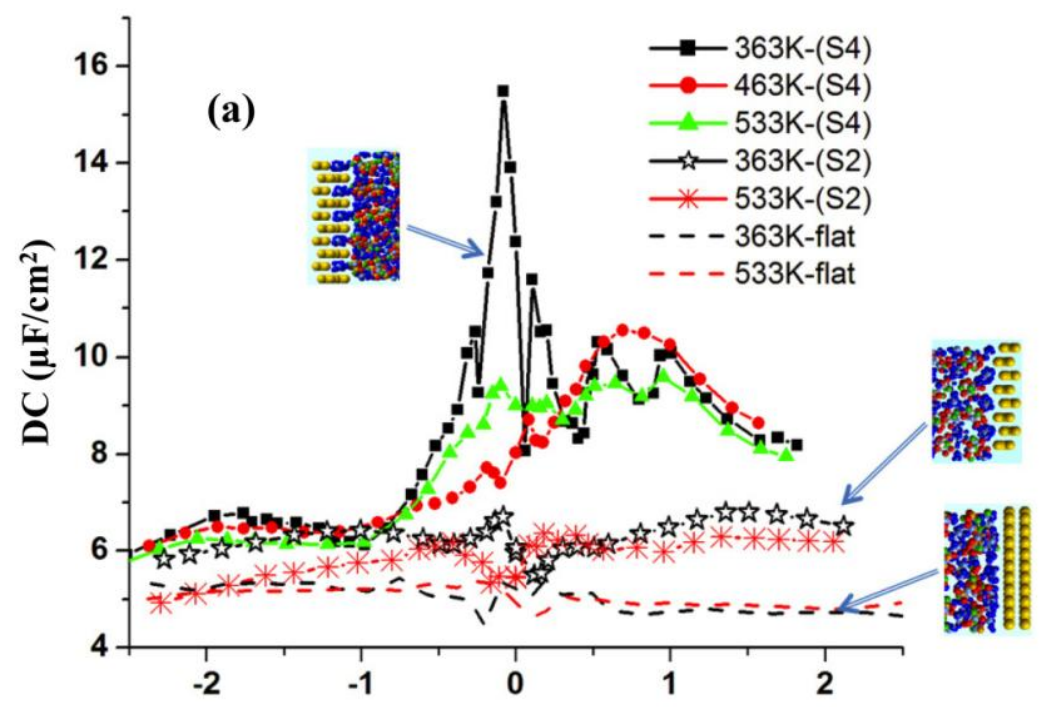

Electrode potential (V)

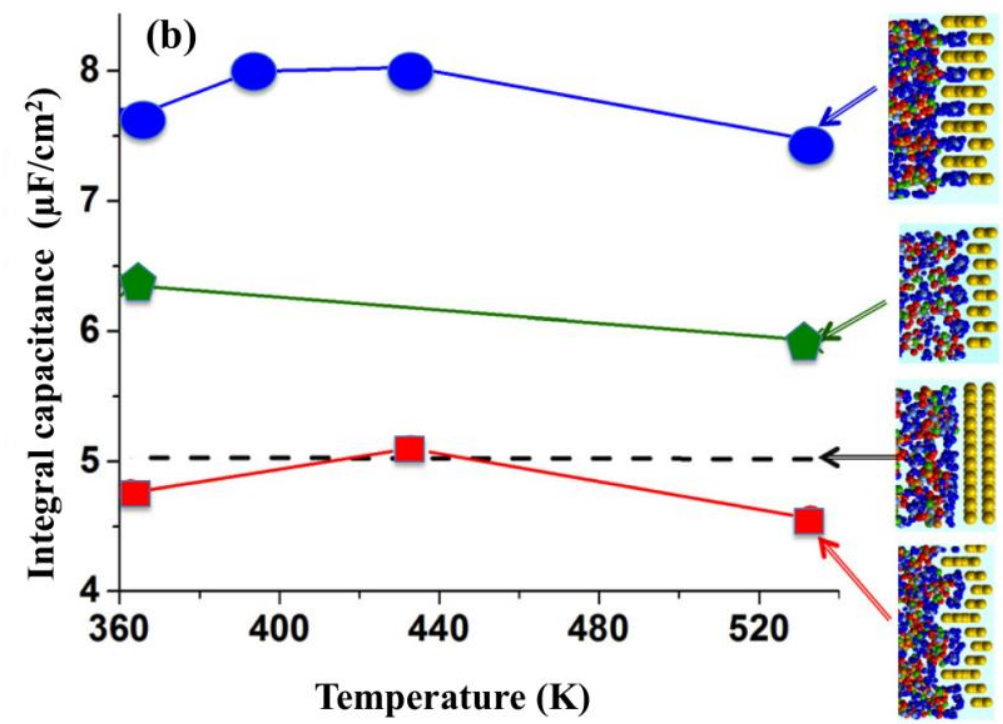

Figure 10. a) The differential capacitance as obtained at several temperatures on electrode surfaces with different surface topography in contact with [Pyr $\left.\left.{ }_{13}\right][F S I] R T I L . b\right)$ Temperature dependence of the integral capacitance for different electrode surfaces. 